\title{
Composite slab numerical strength test method under partial connection approach
}

\begin{abstract}
The present uneconomic strength determination approach for profiled composite slab (PCS) constitutes a serious challenge that contributed significantly to design conservatism. This study seeks to address this challenge by developing and a subsequent experimental validation of a numerical strength determination function for PCS through implementing a rationalbased approach. Hence, a procedural algorithm lead to the development of PCS determination function using longitudinal shear estimation method by considering section slenderness and deck characteristics. The strength test performance between the developed scheme and the experiment-based test results indicates high similarity, demonstrating the viability of the proposed strength determination methodology developed.
\end{abstract}

Keyword: Composite slab; Strength load; Longitudinal shear 